\title{
RETRACTED ARTICLE: Image quality tendency modeling by fusing multiple visual cues
}

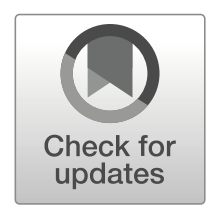

\author{
Yiyang Yao ${ }^{1} \cdot$ Tengfei $\mathrm{Wu}^{2} \cdot$ Jun $\mathrm{Li}^{2}$
}

Received: 30 January 2017 / Revised: 26 April 2017 / Accepted: 4 May 2017 /

Published online: 1 June 2017

(C) Springer Science+Business Media New York 2017

The Editor-in-Chief has retracted this article [1], which was published as part of special issue "Data Security in Multimedia Modeling", because the article shows substantial text overlap most notably with the articles cited [2, 3, 4]. In addition, there is evidence of figure duplication without appropriate permission, as well as evidence suggesting authorship manipulation and an attempt to subvert the peer review process.

The authors have not responded to correspondence about this retraction.

\section{References}

1. Yao, Y., Wu, T. \& Li, J. Image quality tendency modeling by fusing multiple visual cues. Multimed Tools Appl (2017). https://doi.org/10.1007/s11042-017-4821-8

2. Zhang, L. \& Zimmermann, R. Flickr circles: Mining socially-aware aesthetic tendency. IEEE International Conference on Multimedia and Expo (2015). https://doi.org/10.1109 /ICME.2015.7177384

3. Hong, R., Zhang, L., Zhang, C. et al. Flickr circles: Aesthetic tendency discovery by multi-view regularized topic modeling. IEEE Transactions on Multimedia (2016). https://doi.org/10.1109/TMM.2016.2567071

4. Zhang, H., Edwards, R. \& Parker, L. Regularized probabilistic latent semantic analysis with continuous observations. 2012 11th International Conference on Machine Learning and Applications (2013). https://doi.org/10.1109/ICMLA.2012.102

Electronic supplementary material The online version of this article (https://doi.org/10.1007/s11042-0174821-8) contains supplementary material, which is available to authorized users.

Tengfei Wu

zglumg@gmail.com

Yiyang Yao

yiyangyao@gmail.com

Jun Li

junli1778@gmail.com

1 State Grid Zhejiang Electric Power Company Information \& Telecommunication Branch, Hangzhou, China

2 National University of Singapore, Singapore 91121, China 